\title{
Avaliação de Transtornos da Personalidade em Moradores de Rua
}

Assessment of Personality Disorders in Street Dwellers

Evaluación de los Trastornos de Personalidad dn Personas sin Hogar

José Maria Montiel \&

Daniel Bartholomeu

Centro Universitário Fundação Instituto de Ensino para Osasco

Lucas de Francisco Carvalho

\& Fernando Pessotto

Universidade São Francisco

http://dx.doi.org/10.1590/1982-370301992013 
Resumo: Este estudo teve com objetivo comparar a prevalência de tendências de personalidade patológicas entre moradores de rua, comparando-as com outros grupos. Foram participantes do estudo três grupos agrupados em moradores de rua, psiquiátricos e não psiquiátricos (universitários). Foi instrumento de coleta de dados, o Inventário Dimensional dos Transtornos da Personalidade (IDTP). Este instrumento possibilitou uma avaliação do perfil das tendências dos transtornos da personalidade. Entre os resultados encontrados, destaca-se a elevação em determinadas escalas do IDTP, sugerindo uma tendência para desconfiança, impulsividade, necessidade de atenção e comportamentos excêntricos na amostra de moradores de rua. Como considerações, apesar de suas limitações quanto ao número de participantes restrito a uma região do país, e delineamento correlacional, que não permitem inferências causais mais seguras, considera-se a importância de novas investigações acerca do problema de saúde mental do morador de rua visando demarcar este campo de atuação de modo que novas políticas públicas baseadas em evidências possam ser traçadas para esta problemática.

Palavras-chave: Psicodiagnostico. Transtornos mentais. Situação de risco. Saúde. Política Pública.

\begin{abstract}
This study aimed to compare the prevalence of pathological personality tendencies among the homeless and compare these to other groups. Study participants were assigned to three groups: homeless, psychiatric, and non-psychiatric (university). The groups were assessed as per the dimensional inventory of personality disorders (DIPD), which allowed an assessment of the trends in personality disorders. Among the findings, there was an increase in certain DIPD scales that suggested tendencies for distrust, impulsivity, a need for attention, and eccentric behavior in the homeless sample. Despite its limited number of participants restricted to one region of the country and its correlational design, which does not allow causal inferences, this study revealed the importance of further research on mental health problems in the homeless so that new evidence-based public policies can be created for addressing them.
\end{abstract}

Keywords: Psychodiagnostic. Mental disorders. Situation risk. Health. Public Policy.

Resumen: Este estudio tuvo como objetivo comparar el predominio de las tendencias de personalidad patológica entre las personas sin hogar, en comparación con otros grupos. Los participantes del estudio fueron agrupados en tres grupos de personas: sin hogar, psiquiátricos y no psiquiátricos (universidad). El Inventario de Dimensiones de Trastornos de la Personalidad (IDTP) se utilizó como instrumento de recolección de datos. Este instrumento permitió una evaluación del perfil de las tendencias en los trastornos de personalidad. Entre los resultados, se observó un aumento en ciertas escalas del IDTP, lo que sugiere una tendencia a la desconfianza, la impulsividad, la necesidad de atención y comportamiento excéntrico en la muestra de personas sin hogar. Como contrapartida, a pesar de las limitaciones en el número de participantes, restringidos a una región del país, y el diseño de correlación, que no permite inferencias causales más seguras, se considera importante la investigación sobre el problema de salud mental de búsqueda de personas sin hogar como campo a ser considerado en las nuevas políticas públicas basadas en evidencias del problema.

Palavras clave: Psicodiagnóstico. Trastornos mentales. Situación riesgo. Salud. Políticas Públicas.

\section{Introdução}

Muitos fatores podem levar uma pessoa a morar na rua, dentre eles, o rompimento de vínculos afetivos e familiares, problemas psicológicos, abandono ou morte de um ente querido, falta de um núcleo familiar, situação econômica resultante de perda do emprego ou mesmo a dificuldade para se inserir no mercado de trabalho (Neves, 1995). Castel (1998) sintetiza que esse quadro relaciona-se com situação extrema de ruptura de relações familiares e afetivas, além de ruptura total ou parcial com o mercado de trabalho e da não participação social efetiva, acarretando assim a exclusão social. Silva (2006) complementa que essa população, mesmo sendo 
um grupo heterogêneo, possui características semelhantes como a condição de pobreza, vínculos familiares prejudicados, inexistência de moradia regular, entre outros.

Uma multiplicidade de termos caracteriza sujeitos na vivência de rua, como mendigo, sofredor de rua, sem teto, pedinte, indigente, excluído, morador de rua, entre outros, desqualificando, muitas vezes, as pessoas que, por motivos diversos, se encontram nessa situação. Considerando a vivência na rua como processual, ou seja, um período sem caráter necessariamente crônico, são encontrados textos na literatura que adotam o termo "pessoa em situação de rua" (Giorgetti, 2006), mas é bastante recorrente o uso do termo "moradores de rua". Por isso, para o presente estudo ambos os termos foram utilizados. Ao lado disso, Ferreira (2006) propõe outro tipo de classificação, considerando outras variáveis, a saber, a violência, drogas, desemprego e problemas de saúde. No grupo de violência, estão inseridos os casos relacionados à violência doméstica, sejam física ou psicológica, preconceitos relacionadas às pessoas de baixa renda que, por vez, mesmo não tendo meios de autossustento, prefere sair de casa, ocasionando um longo período de rompimento familiar ou mesmo extinguindo os vínculos afetivos. No segundo grupo, referente às drogas, muitas pessoas em situação de rua, fazem uso abusivo do álcool e outros tipos de droga sendo a rua a porta da liberdade para manutenção do vício, tendo em vista que os mesmos não sofrem o controle da família e até mesmo de albergues, reforçando, portanto, a desvinculação dos laços familiares. O autor destaca o desemprego como formação do terceiro grupo, incluindo nele os migrantes e ex-detentos que não conseguem colocação no mercado de trabalho. O quarto grupo está relacionado aos sujeitos que possuem problemas de saúde, encaixando-se os que apresentam algum sofrimento psíquico, como também os egressos de clínicas ou hospitais públicos de tratamento psiquiátrico que acabam voltando para a situação de rua.

Além do sofrimento físico que esta população está submetida, alguns autores (Botti,
Castro, Silva, Silva, Oliveira et al., 2010; Caton, Hasin, Shrout, Opler, Hirshfield et al., 2000; Fazel, Khosia, Doll \& Geddes, 2008; Rosa, Secco \& Brêtas, 2006) salientam ainda a existência de transtornos mentais, sem, contudo, identificar se estão na etiologia ou manutenção dessa condição. Pesquisas americanas com moradores de rua indicam que cerca de $90 \%$ destas pessoas receberam um diagnóstico psiquiátrico, sendo aproximadamente $40 \%$ de quadros de psicose e $29 \%$ uso abusivo de álcool (Bassuk, Rubin \& Lauriat, 1984). No Reino Unido, a população de rua é caracterizada em seus 31\% por diagnóstico de esquizofrenia (Timms \& Fry, 1989), sendo ainda $50 \%$ com qualquer outro diagnóstico do eixo l/transtornos clínicos e $70 \%$ do eixo II/transtornos da personalidade (Drake, Yovetich, Bebout, Harris \& McHugo, 1997). Esses autores sugerem que moradores de rua podem conseguir emprego desde que sua condição de saúde seja adequadamente tratada, dando atenção para a saúde mental destes sujeitos como condição para que a reincidência não venha a ocorrer. Nesses termos, o problema do morador de rua não pode ser tratado pela providência de acomodações ou suprimento de necessidades básicas somente, mas com um cuidado mais detido de sua condição de saúde.

Sobre a prevalência de transtornos de personalidade em moradores de rua, Fazel et al., (2008) descrevem uma taxa de $70 \%$ de prevalência desses transtornos sendo mais frequentes tendências esquizoides, borderline, dependente e antissocial. O transtorno antissocial recebeu a maior atenção nessas pesquisas com taxas de 10 a $40 \%$, o que foi também constatado por Caton, Hasin, Shrout, Opler, Hirschfield et al. (2000). Os autores enfatizam que resta estudar se esses transtornos estão na etiologia ou manutenção desse problema social. Além disso, constam dentre as sugestões para o desenvolvimento de estratégias de manejo clínico destes problemas técnicas de regulação emocional, treinamento para solução de problemas, treinamento para habilidades interpessoais, dentre outras, que são especialmente terapêuticos em jovens em situação de prevenção de desregulagem afetiva e condutas disruptivas. Neste sentido, acerca da personalidade, deve-se considerar 
que seus diferentes modos podem estar relacionados à experiência de vida de cada pessoa, da capacidade que esta possui para lidar com os eventos estressores da vida, podendo desenvolver-se de maneira mais adaptativa ou não. Segundo Millon (2011), o estilo mal adaptativo de vida apresentado por uma pessoa, pode estar ligado à maneira como ela se vê no mundo e ainda como percebe o mundo em si, em seu modo de expressar as emoções e comportar-se socialmente. Quando estes se apresentam instáveis e danosos, tendencialmente afetará várias áreas de vida, assim como também, aos que com ele convivem. Sendo assim, constituirse-ão em transtornos da personalidade.

Na proposta de Millon (1990, 2011), a personalidade é considerada como uma organização ativa dos traços ligados intrinsecamente ao self, compostos a partir de características genéticas herdadas, de singularidades vivenciadas na existência, percepção de ser e estar no mundo e do mundo em si, fazendo cada indivíduo único em seu estilo de pensar, ser, sentir e agir em diversos papéis sociais. Os traços de personalidade são padrões duradouros de percepção, interação e pensamentos acerca de si próprio e do ambiente, apresentados em uma ampla gama de contextos sociais e pessoais. Quando estes se apresentam inflexíveis e mal adaptativos, causando expressivo comprometimento no desempenho de uma pessoa, originam os transtornos de personalidade (American Psychiatry Association, 2002).

Para Millon, (1990) os transtornos de personalidade desenvolvem-se num continuum de tempo, sofrem influência tanto biológica como do ambiente e algumas pessoas adquirem hábitos e atitudes caracterizadas por falta de resiliência, que se instalam automaticamente e vão transformando a experiência de vida dessas pessoas. Em consonância com a última versão do Manual Estatístico e Diagnóstico em Transtornos Mentais ([DSM-5]; APA, 2013), na teoria de Millon os transtornos da personalidade podem ser identificados na população pela presença estável de manifestações patológicas em dimensões da personalidade e seus traços, envolvendo prejuízos interpessoais e na relação com o eu/self (Millon, 2011; Skodol, Clark, Bender, Krueger, Livesley et al., 2011). Na busca por uma melhor compreensão da personalidade e transtornos relacionados, Millon apresenta uma teoria contemporânea em que integra vários conceitos, definindo a personalidade como um complexo padrão de características psicológicas, intrinsecamente arraigadas, com elementos inconscientes que se expressam automaticamente em todo conjunto. Essas características intrínsecas emergem de um complexo conjunto de disposições biológicas, da aprendizagem e experiências do indivíduo, que constituem padrões distintos de percepções, sentimentos, pensamentos, da resiliência e dos comportamentos apresentados por um indivíduo (Millon, 1990, 1999; Millon \& Davis, 1996; Millon, Everly \& Davis, 1995; Millon, Grossman, Millon, Meagher \& Ramanath, 2004).

Os transtornos de personalidade são descritos no DSM-IV-TR (APA, 2002), especificamente no eixo II, e compreendem os Transtornos de Personalidade Paranoide, Esquizoide, Esquizotípico, Antissocial, Borderline, Histriônico, Narcisista, Evitativo, Dependente, ObsessivoCompulsivo e Sem Outra Especificação. Desta maneira, o modelo multiaxial confirma sua utilização para diagnosticar, levando em consideração que o paciente é mais do que a soma do diagnóstico. Vale ressaltar que essa mesma classificação diagnóstica está presente na seção 2 do DSM-5 (APA, 2013). Quando se considera os sintomas de um paciente em relação às suas características mais profundas, obtêm-se compreensão de sintomas que mais afetam a pessoa e os traços de personalidade considerados em separado (Millon, Grossman, Millon, Meagher \& Ramnath, 2004). Ainda, aponta-se que os transtornos de personalidade são assim considerados desde que o modo de funcionamento do indivíduo esteja atrelado a decréscimos dos funcionamentos social do indivíduo, o que, em situação de moradia de rua fica bastante evidente, já que essas pessoas, como já mencionado, não possuem emprego fixo, moradia fixa, sendo considerados excluídos socialmente (APA, 2002). 
Analisar a prevalência desses transtornos em moradores de rua assume relevância, já que favoreceriam a identificação de aspectos que podem estar no cerne destes prejuízos sociais vivenciados por estes sujeitos. Além disso, a caracterização das tendências patológicas de personalidade de moradores de rua pode facilitar o delineamento de intervenções com estas pessoas, já que, por um lado, caracterizaria o problema como de saúde mental, possibilitando o melhor direcionamento de políticas públicas nesta direção e, por outro, considerando a personalidade em seu aspecto funcional e dimensional e que, portanto, sua análise deva envolver os modos de cognição, regulação afetiva, entre outras características, englobaria uma gama de aspectos que seriam úteis no delineamento de intervenções nesta área (Bartholomeu, Montiel, Silva, Cozza \& Machado, 2013; Millon \& Davis, 1996; Westen \& Bradley, 2005; Widiger \& Clark, 2000). Em que pese isso tudo, no Brasil, observa-se também uma carência de literatura no que se refere à epidemiologia de saúde mental em moradores de rua (Botti et al., 2010). De fato, problemas de álcool tendem a ser os mais indicados, neste particular, na maior parte dos trabalhos, como de Botti (2009), Botti et al. (2010), Rosa et al. (2006), e que também enfatizam a epidemiologia dos problemas de saúde mental em população de rua, bem como relacionam a saúde mental como parte da adaptação social do individuo, sobressaltando aspectos sociais dos transtornos mentais.

Os trabalhos realizados em Belo Horizonte foram bastante interessantes neste aspecto, na medida em que se estabeleceu uma parceria entre a Secretaria de Saúde e Assistência Social para instalar uma política pública para lidar com o problema do morador de rua, enfatizando a interface entre a saúde mental e a Psicologia Social. Apesar disso, não existem estudos empíricos que estabeleçam essas relações entre adaptação social e os problemas de saúde mental do morador de rua, sendo uma lacuna na literatura. Ainda, pesquisas tendem a manter o foco sobre a inclusão social do morador de rua, considerando esta como relacionada à saúde mental destes indivíduos, o que se opõe, em certa medida, às sugestões de Drake et al.
(1997) e Fazel et al. (2008) em que se sugere que um tratamento da condição de saúde destas pessoas deva ser efetuado para então se trabalhar a inclusão social destas pessoas. Neste contexto, não se encontram, no Brasil, trabalhos com ênfase nos transtornos de personalidade em moradores de rua, tornando pertinente a execução de pesquisas neste âmbito. Com isso, o objetivo deste estudo foi comparar a prevalência de tendências de personalidade patológicas tal como consideradas no DSM-IV entre moradores de rua, um grupo de pacientes psiquiátricos e universitários (grupo controle).

\section{Método}

\section{Participantes}

Para este estudo foram coletados dados com três grupos de participantes, agrupados em moradores de rua, psiquiátricos e não psiquiátricos. Os dois últimos grupos retratam a mesma amostra utilizada na pesquisa de Carvalho (2008). A pesquisa foi realizada com 80 pessoas em situação de rua, variando entre 18 e 68 anos $(M=34)$; $(D P=11,17)$, sendo $90,7 \%$ do sexo masculino e $9,3 \%$ do sexo feminino. Dos 54 participantes, 29,6\% fizeram tratamento psiquiátrico (antes do período da coleta de dados) e 1,9\% ainda faziam (no período da coleta) e foram excluídos da amostra. Do total dos participantes, 68,5\% não faziam tratamento psicológico (antes do período da coleta de dados) e 96,3\% do total nunca fizeram, apenas $3,7 \%$ desses faziam (no período da coleta) e também foram excluídos da amostra, restando 72 participantes. Os participantes da amostra moradores de rua que relataram realizar tratamento psiquiátrico ou psicológico foram excluídos já que poderiam ser confundidos com amostra clínica. Em relação aos que fizeram "tratamento psicológico ou psiquiátrico", a frequência mínima de "tempo" para um mês, como para frequência de quatro meses, a resposta foi de $5,6 \%(N=54)$, para cada período de tempo. Já para os que "não lembram" a resposta foi de $7,4 \%(N=54)$. Do total de 72 participantes para o "consumo de remédio" obtevese resposta positiva para $9,3 \%$ e $90,7 \%$ de resposta negativa para consumo de remédio 
do total de participantes. Quanto ao grau de escolaridade, todos os moradores de rua obtiveram ensino fundamental incompleto.

A maioria dos participantes morava nas ruas porque saíram de casa fugidos, dadas as condições familiares precárias que envolviam abusos e maus tratos $(66 \%)$ ou porque foram expulsos de seus lares pelo uso de drogas ou condutas antissociais (34\%). Todos os participantes eram atendidos em uma instituição (abrigo) que fornecia alimento (café da manhã e almoço), roupa, banho e cuidados de higiene e dormitório para pessoas em situação de rua e migrantes. A instituição não apresentava psicólogo em sua grade de funcionários, somente assistentes sociais que faziam encaminhamentos, em sua maioria para outros órgãos públicos visando fornecer passagens de ônibus ou encaminhamento para empregos. No momento da aplicação, todos os moradores de rua estavam desempregados.

Em relação ao grupo de pacientes psiquiátricos $(\mathrm{N}=74)$, a idade variou entre $18 \mathrm{e}$ 67 anos $(M=37,85 ; D P=13,04)$, sendo 54 $(73 \%)$ mulheres. A escolaridade desse grupo variou entre ensino fundamental incompleto e ensino superior completo. Todos os sujeitos desse grupo responderam afirmativamente em relação a realizar tratamento psiquiátrico e fazer uso de medicação, sendo que 56 deles tinham diagnóstico conhecido. Os detalhes acerca do diagnóstico desses pacientes estão descritos na literatura (Carvalho, 2008). No que se refere ao grupo de não pacientes $(\mathrm{N}=250)$, todos responderam negativamente sobre realizar tratamento psiquiátrico ou fazer uso de medicação, sendo todos universitários, entre 18 e 67 anos $(M=23,40$; $D P=6,18)$, distribuídos entre homens $(\mathrm{N}=74)$ e mulheres $(\mathrm{N}=176)$.

\section{Instrumento}

Foi utilizado como instrumento de coleta de dados, o Inventário Dimensional dos Transtornos da Personalidade (IDTP). Optouse por este devido a ser um instrumento validado, de acordo com a cultura brasileira (Carvalho, 2008). Este instrumento possibilitou uma avaliação do perfil das tendências dos transtornos da personalidade em pessoas na situação de rua. O IDTP é um instrumento para avaliar os transtornos da personalidade, composto por 100 itens, distribuídos em 15 escalas distintas: Depressivo, Esquizoide, Borderline, Paranoide, Sádico, Compulsivo, Masoquista, Antissocial, Dependente, Histriônico, Negativista, Esquizotípico, Evitativo, Narcisista e de Validade e Desejabílidade Social. Para este estudo, foram consideradas somente as quatorze escalas que avaliam transtornos da personalidade. As respostas aos itens foram disponibilizadas em escala Likert de quatro pontos, onde: (1) "Não me descreve", (2) "Me descreve pouco"; (3) "Me descreve muito" e (4) "Me descreve extremamente ou totalmente". No cabeçalho obtêm-se as seguintes informações: iniciais do nome, data de nascimento, e-mail, sexo, se este realiza ou já realizou tratamento psicológico ou psiquiátrico se sim quanto tempo. Os escores foram obtidos através da fatoração bruta das respostas apresentadas.

\section{Procedimentos}

Foi entregue aos participantes o Termo de Consentimento Livre e Esclarecido onde consta o objetivo principal deste estudo, como também informações aos participes acerca da divulgação dos resultados de acordo com as normas éticas que regem este projeto. Posteriormente ao consentimento e assinatura deste, foi aplicado o inventário. Cabe mencionar que a pesquisa teve aprovação do Comitê de Ética da Anhanguera Educacional, sob o protocolo $\mathrm{n}^{\circ}$ 1240/2011. Optou-se em aplicar o inventário de forma individual, ou seja, assistida, por se tratar de um grupo que apresenta sujeitos com baixo grau de escolaridade, dentre outros fatores ligados à dificuldade de compreensão, relacionada ao uso de substâncias lícitas como o "álcool" ou ilícitas, assim como a cocaína, craque, maconha, dentre outros. Nenhum dos sujeitos estava sob efeito de quaisquer tipos de substâncias no momento da aplicação do instrumento. Devido a estes fatores, o tempo de aplicação foi superior ao tempo estimado de 20 minutos. Os participantes que 
foram convidados a responder a pesquisa são assistidos por uma instituição assistencialista localizada no interior do estado de São Paulo. Em relação aos demais grupos, os procedimentos para coletas de dados apresentados de maneira detalhadas encontram-se em Carvalho (2008).

\section{Resultados}

Visando uma verificação e rastreio inicial da amostra presentemente estudada, inicialmente foram calculados os dados descritivos dos sujeitos nas 14 escalas do IDTP. Os dados estão apresentados na tabela 1. Pode-se observar que a variância nas escalas ficou próxima à máxima em ambas as extremidades para todos os casos, sendo que a escala Antissocial foi a com menor variância. Em relação à média, a menor foi obtida pela escala Sádico e a maior pela escala Compulsivo. Além disso, as médias tenderam a ficar próximas ou acima da tendência central $(2,5$; isto é, o ponto no qual a escala apresenta a mesma distância do ponto mínimo, no caso 1 , e do ponto máximo, no caso 4), sugerindo direção à concordância aos itens do instrumento, e a variância ao redor da média ficou entre 0,56 (Paranoide) e 0,75 (Esquizotípico).
Ainda na tabela 1, estão apresentas entre parênteses as médias obtidas no estudo de Carvalho (2008), de modo que a penúltima coluna $(p)$ refere-se à significância da comparação entre os dados presentemente relatados e os dados relatados por Carvalho (2008), e a última coluna ao tamanho do efeito dessa comparação. Pode-se notar que em todos os casos a média da presente amostra foi maior, $\mathrm{e}$ no geral, de maneira expressiva (e significativa para todas as escalas), o que pode ser verificado pela medida de tamanho do efeito, $d$ de Cohen. Na continuidade, de acordo com os objetivos deste estudo, foram realizadas primeiramente análises investigando a estrutura e consistência interna do IDTP e suas escalas. $\mathrm{Na}$ tabela 2 estão apresentados os índices de consistência interna ( $\alpha$ de Cronbach) das escalas do instrumento, bem como os índices obtidos por Carvalho (2008).

Em quase todos os casos, o índice encontrado para as escalas com base na presente amostra foi inferir ao relatado por Carvalho (2008), especialmente para as escalas Borderline, Paranoide, Antissocial, Histriônico e Negativista. Considerando uma importante característica da amostra desta pesquisa, qual seja moradores de rua, todos com nível de escolaridade não superior ao ensino

Tabela 1. Dados descritivos da amostra nas escalas do IDTP.

\begin{tabular}{lcccccc}
\hline Escalas & Mínimo & Máximo & Média & DP & P & d \\
\hline Depressivo & 1,00 & 4,00 & $2,39(1,54)$ & 0,73 & 0,001 & 1,30 \\
Esquizoide & 1,00 & 3,63 & $2,19(1,55)$ & 0,66 & 0,001 & 1,15 \\
Borderline & 1,57 & 4,00 & $2,84(2,46)$ & 0,60 & 0,001 & 0,62 \\
Paranoide & 1,00 & 3,67 & $2,65(1,84)$ & 0,56 & 0,001 & 1,43 \\
Sádico & 1,00 & 3,50 & $1,84(1,52)$ & 0,69 & 0,001 & 0,60 \\
Compulsivo & 1,40 & 4,00 & $3,07(2,76)$ & 0,69 & 0,001 & 0,46 \\
Masoquista & 1,00 & 3,86 & $2,37(1,59)$ & 0,69 & 0,001 & 1,34 \\
Antissocial & 1,14 & 3,57 & $2,33(1,72)$ & 0,58 & 0,001 & 1,10 \\
Dependente & 1,00 & 4,00 & $1,92(1,56)$ & 0,66 & 0,001 & 0,65 \\
Histriônico & 1,00 & 3,80 & $2,20(1,73)$ & 0,68 & 0,001 & 0,78 \\
Negativista & 1,00 & 4,00 & $2,24(1,46)$ & 0,66 & 0,001 & 1,56 \\
Esquizotípico & 1,00 & 4,00 & $2,57(1,79)$ & 0,75 & 0,001 & 1,14 \\
Evitativo & 1,00 & 4,00 & $2,46(1,79)$ & 0,69 & 0,001 & 1,09 \\
Narcisista & 1,25 & 3,88 & $2,45(2,18)$ & 0,59 & 0,001 & 0,49 \\
\hline Dados de signifiann
\end{tabular}

Dados de significância calculados via teste t para uma só amostra. 
fundamental, pode-se inferir a possibilidade de dificuldade de compreensão em leitura por alguns dos participantes, o que pode impactar nos índices evidenciados. Além disso, como a diminuição dos índices foi mais evidente em determinadas escalas, pode-se também hipotetizar que as características típicas nessas escalas tenham sido atípicas para a amostra, o que potencializou a dificuldade de compreensão. Contudo, essas são hipóteses que não foram verificadas no estudo. Na sequência, na tabela 3 , estão apresentados os dados referentes à ANOVA, comparando as médias da amostra de moradores de rua com as amostras utilizadas por Carvalho (2008), isto é, pacientes com diagnóstico psiquiátrico e pessoas sem diagnóstico psiquiátrico conhecido.

Em todos os casos de comparação, as médias da amostra de moradores de rua foram superiores às médias dos outros grupos, e também significativas em todos os casos. Contudo, nem sempre os três grupos se distinguiram, tal qual verificado pela análise post hoc. $\mathrm{O}$ número de grupos distintos pode ser visualizado na última coluna da tabela 3, pela qual observa-se que sete escalas apresentaram

Tabela 2. Índices de consistência interna das escalas do IDTP.

\begin{tabular}{lcc}
\hline Escalas & \multicolumn{2}{c}{$\alpha$ de Cronbach } \\
\hline & $\begin{array}{c}\text { Presente } \\
\text { estudo }\end{array}$ & $\begin{array}{c}\text { Carvalho } \\
(2008)\end{array}$ \\
Depressivo & 0,75 & 0,89 \\
Esquizoide & 0,72 & 0,86 \\
Borderline & 0,58 & 0,79 \\
Paranoide & 0,46 & 0,77 \\
Sádico & 0,71 & 0,76 \\
Compulsivo & 0,70 & 0,77 \\
Masoquista & 0,71 & 0,81 \\
Antissocial & 0,48 & 0,73 \\
Dependente & 0,66 & 0,77 \\
Histriônico & 0,54 & 0,71 \\
Negativista & 0,58 & 0,74 \\
Esquizotípico & 0,83 & 0,76 \\
Evitativo & 0,72 & 0,81 \\
Narcisista & 0,63 & 0,65 \\
\hline
\end{tabular}

distinção entre os três grupos. Outras sete escalas apresentaram diferença significativa somente em dois grupos, sendo, Borderline (não psiquiátricos x psiquiátricos e moradores de rua), Sádico (não psiquiátricos x psiquiátricos e moradores de rua), Compulsivo (moradores de rua $x$ não psiquiátricos $\mathrm{e}$ psiquiátricos), (moradores de rua $x$ não psiquiátricos e psiquiátricos), Dependente (moradores de rua $x$ não psiquiátricos $\mathrm{e}$ psiquiátricos), Histriônico (moradores de rua x não psiquiátricos e psiquiátricos) e Narcisista (moradores de rua $x$ não psiquiátricos e psiquiátricos). Complementando a ANOVA, os perfis das três amostras de acordo com as 14 dimensões do IDTP foram comparados via análise de perfis por medidas repetidas. A comparação foi significativa ( $F=10,908$; $p<0,001)$ e os perfis podem ser visualizados na Figura 1.

Por meio da figura é possível notar uma clara evolução em termos de concordância aos itens das escalas do IDTP, isto é, a amostra que tendeu a concordar menos com os itens foi a não psiquiátrica, seguida pela psiquiátrica e, por último, o grupo com maior tendência a concordar foi o de moradores de rua. Para

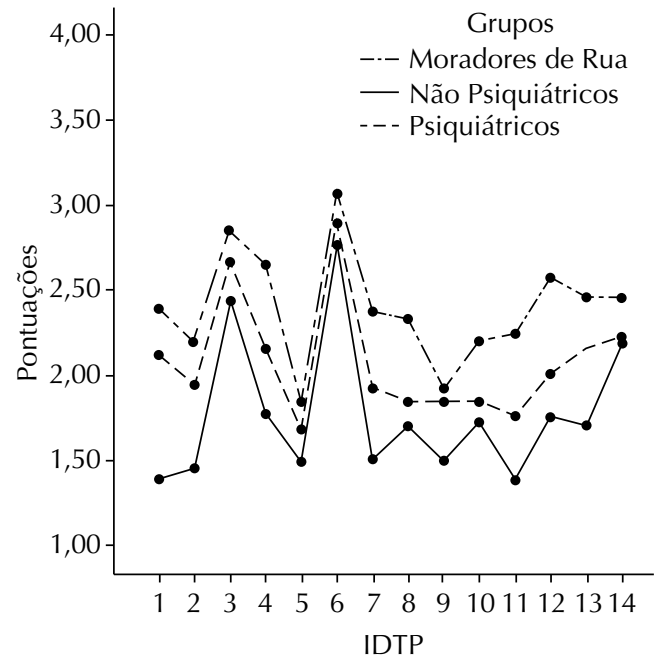

1 = Depressivo; 2 = Esquizoide; 3 = Borderline; 4 = Paranoide; 5 = Sádico; 6 = Compulsivo; 7 = Masoquista; 8 = Antissocial; 9 = Dependente; 10 = Histriônico; 11 = Negativista; 12 =Esquizotípico; 13 =Evitativo; $14=$ Narcisista.

Figura 1. Comparação entre os perfis no IDTP. 
Tabela 3. Comparação entre as médias das amostras nas escalas do IDTP.

\begin{tabular}{|c|c|c|c|c|c|c|c|}
\hline Escalas & Grupos & Média & DP & $\mathbf{N}$ & $\mathbf{F}$ & $\mathbf{P}$ & Grupos distintos \\
\hline \multirow[t]{3}{*}{ Depressivo } & MR & 2,3 & 0,73 & 71 & 94,002 & 0,001 & 3 \\
\hline & NP & 1,3 & 0,48 & 247 & & & \\
\hline & Psi & 2,1 & 0,80 & 72 & & & \\
\hline \multirow[t]{3}{*}{ Esquizoide } & $M R$ & 2,1 & 0,66 & 71 & 59,463 & 0,001 & 3 \\
\hline & NP & 1,4 & 0,43 & 247 & & & \\
\hline & Psi & 1,9 & 0,75 & 72 & & & \\
\hline \multirow[t]{3}{*}{ Borderline } & MR & 2,8 & 0,60 & 71 & 13,664 & 0,001 & 2 \\
\hline & NP & 2,4 & 0,57 & 247 & & & \\
\hline & Psi & 2,6 & 0,73 & 72 & & & \\
\hline \multirow[t]{3}{*}{ Paranoide } & MR & 2,6 & 0,56 & 71 & 72,468 & 0,001 & 3 \\
\hline & NP & 1,7 & 0,47 & 247 & & & \\
\hline & Psi & 2,1 & 0,76 & 72 & & & \\
\hline \multirow[t]{3}{*}{ Sádico } & $M R$ & 1,8 & 0,69 & 71 & 12,794 & 0,001 & 2 \\
\hline & NP & 1,4 & 0,46 & 247 & & & \\
\hline & Psi & 1,6 & 0,64 & 72 & & & \\
\hline \multirow[t]{3}{*}{ Compulsivo } & MR & 3,0 & 0,69 & 71 & 5,891 & 0,003 & 2 \\
\hline & NP & 2,7 & 0,64 & 247 & & & \\
\hline & Psi & 2,8 & 0,75 & 72 & & & \\
\hline \multirow[t]{3}{*}{ Masoquista } & MR & 2,3 & 0,69 & 71 & 66,129 & 0,001 & 3 \\
\hline & NP & 1,5 & 0,50 & 247 & & & \\
\hline & Psi & 1,9 & 0,66 & 72 & & & \\
\hline \multirow[t]{3}{*}{ Antissocial } & MR & 2,3 & 0,58 & 71 & 36,854 & 0,001 & 2 \\
\hline & NP & 1,7 & 0,48 & 247 & & & \\
\hline & Psi & 1,8 & 0,67 & 72 & & & \\
\hline \multirow[t]{3}{*}{ Dependente } & MR & 1,9 & 0,66 & 71 & 22,333 & 0,001 & 2 \\
\hline & NP & 1,5 & 0,48 & 247 & & & \\
\hline & Psi & 1,8 & 0,63 & 72 & & & \\
\hline \multirow[t]{3}{*}{ Histriônico } & MR & 2,2 & 0,68 & 71 & 17,197 & 0,001 & 2 \\
\hline & NP & 1,7 & 0,55 & 247 & & & \\
\hline & Psi & 1,8 & 0,71 & 72 & & & \\
\hline \multirow[t]{3}{*}{ Negativista } & MR & 2,2 & 0,66 & 71 & 86,200 & 0,001 & 3 \\
\hline & NP & 1,3 & 0,36 & 247 & & & \\
\hline & Psi & 1,7 & 0,65 & 72 & & & \\
\hline \multirow[t]{3}{*}{ Esquizotípico } & MR & 2,5 & 0,75 & 71 & 38,886 & 0,001 & 3 \\
\hline & NP & 1,7 & 0,64 & 247 & & & \\
\hline & Psi & 2,0 & 0,78 & 72 & & & \\
\hline \multirow[t]{3}{*}{ Evitativo } & MR & 2,4 & 0,69 & 71 & 48,786 & 0,001 & 3 \\
\hline & NP & 1,7 & 0,51 & 247 & & & \\
\hline & Psi & 2,1 & 0,77 & 72 & & & \\
\hline \multirow[t]{3}{*}{ Narcisista } & MR & 2,4 & 0,59 & 71 & 6,644 & 0,001 & 2 \\
\hline & NP & 2,1 & 0,51 & 247 & & & \\
\hline & Psi & 2,2 & 0,62 & 72 & & & \\
\hline
\end{tabular}

$\overline{M R}=$ moradores de rua; $\mathrm{NP}=$ não psiquiátricos; $\mathrm{Psi}=$ pacientes psiquiátricos. 
além disso, a distinção mais clara nos perfis dessa amostra com as demais encontra-se nas escalas Paranoide, Antissocial, Histriônico e Esquizotípico, com pontuações expressivamente maiores neste grupo.

\section{Discussão}

Esta pesquisa foi planejada tendo em vista a carência de estudos no Brasil que tratem dos transtornos de personalidade em moradores de rua. Assim, os resultados evidenciados sugerem alterações das tendências de personalidade destes sujeitos maiores que de pacientes psiquiátricos, indicando a magnitude um tanto exacerbada de seus problemas, já que os pacientes psiquiátricos encontravam-se em tratamento em clínicas especializadas, tendo já algum cuidado sobre sua saúde mental. Neste sentido, os dados sugerem que os problemas de saúde mental relacionados a desvios de personalidade podem estar mais proeminentes em moradores de rua demandando uma maior atenção e cuidado em termos de saúde mental. Tal fato reitera a necessidade urgente de direcionamento de políticas públicas para essa realidade da moradia de rua, tratando esse problema dentro do âmbito da saúde anteriormente ao processo de inclusão social, como já é feito em outros países como Estados Unidos e Reino Unido (Caton et al., 2000; Drake et al., 1997; Fazel et al., 2008).

Seguindo os pressupostos acima, concordase com Caton et al. (2000) na medida em que estes chamam a atenção para que o cuidado com a saúde mental do morador de rua seja primário ao cuidado deste problema social e tem um papel importante na quebra da manutenção deste. Ao lado disso, entende-se que à parte do problema da saúde mental destas pessoas que o presente trabalho desvela, a compreensão dos padrões de funcionamento da personalidade predominantes neste grupo favorece a concepção de como planejar as intervenções neste âmbito a partir de técnicas de regulação emocional, solução de problemas, dentre outras que visam cuidar e prevenir a desregulagem afetiva e condutas disruptivas (Fazel et al., 2008). Apesar de todos os escores em cada um dos transtornos estar acima das médias do grupo clínico e controle, os padrões de personalidade com maiores alterações em moradores de rua em detrimento dos demais grupos estudados foram Paranoide, Antissocial, Histriônico e Esquizotípico. Tal fato corrobora em partes a pesquisa de Fazel et al. (2008) em que os autores também evidenciaram maior prevalência de transtorno antissocial nesta população. Apesar disso, os autores também encontraram predominância em padrões Esquizoides, Borderline e Dependente. De fato, nas tendências de personalidade patológicas Borderline e Sádica, o grupo de moradores de rua igualou-se ao de pacientes psiquiátricos, sendo distintos somente de não psiquiátricos. Nas demais avaliações feitas, os moradores de rua predominaram em detrimento dos outros dois grupos.

Quanto aos funcionamentos de personalidade mais aberrantes nestes sujeitos, o paranoide envolve uma tendência a ser constantemente desconfiado, sendo sujeitos que suspeitam quanto a atitudes, pensamentos e intenções de outros para consigo. Se esforçam para confiar ou ser íntimos de outras pessoas, devido ao medo de confidenciar suas experiências e esse revelação possa tornar alvo de si mesmo. Interpretam de forma errônea situações genuínas ou elogios dirigidos a ele (APA, 2002). Por sua vez, indivíduos acometidos de transtorno antissocial apresentam comportamentos e atos delinquentes, que constituem detenção. Agem de forma impulsiva, dificultando planejar o futuro, ainda que próximo. São pessoas extremamente irritáveis, que facilitam os atos agressivos e lutas corporais. Buscam obter vantagens e prazer em seus empenhos. A personalidade Histriônica é caracterizada por pessoas que gostam de chamar atenção para si e utilizam à aparência física como atrativo. Comportam-se como se estivessem desempenhando papéis em teatro, exagero nas expressões emocionais e dramaticidade, como também repentinas mudanças de expressões emocionais. As interações sociais e profissionais são marcadas por superficialidades e comportamentos sexualmente provocantes ou sedutores. Finalmente, 
tendências esquizotípicas caracterizam pessoas que acreditam ter poderes mágicos, controlam e influenciam o comportamento das pessoas, acreditam ser capazes de ler o pensamento e pressentir os acontecimentos, supersticiosas, interpretam incorretamente os acontecimentos, fantasiando suas ações e ideias, ouvem vozes ou até mesma sentem a presença de outra pessoa, constituindo em "ideação paranoide", discursos vagos ou desconexos, não se sentem à vontade diante dos outros, ficam excessivamente ansiosos, têm dificuldades na formação de vínculos além do grupo primário, são excêntricos e seus comportamentos esquisitos (APA, 2002).

Talvez se possa pensar que, em decorrência das histórias de vida dessas pessoas e a situação de rua, possa ter agravado ou mesmo provocado certas destas tendências, ou mesmo seja resultado delas. Essas são perguntas que devem ser analisadas em estudos posteriores com delineamentos que deem conta de tais aspectos. A teoria de Millon (Millon \& Davis, 1996) sugere também que a etiologia dos transtornos de personalidade está atrelada à história patogênica específica e que condições ambientais tendem a agravar e reiterar as tendências. Todavia, testes para todos os aspectos sugeridos pelo autor ainda não foram feitos, convidando a novas investigações, mas não deixando de ser interessante analisar a situação da moradia de rua por esta perspectiva. Apesar disso, com base nos dados da presente pesquisa, pode-se dizer que a situação de moradia de rua está associada principalmente a essas tendências que, enquanto recursos da personalidade destes indivíduos tendem a favorecer, de certa forma, sua adaptação ao meio em que vivem (Millon, 1999). Sobre as tendências histriônicas e antissociais, Hamburger, Lilienfeld e Hogben (1996) mencionam que se tratam de variações masculinas e femininas das mesmas tendências, devendo ser ponderado também, neste caso, o quanto as tendências à agressividade em suas diferentes formas como meio de se obter gratificações não estão imbricadas ao funcionamento básico deste tipo de sujeito que está em situação de exclusão e vivendo na rua. Tal fato também sugere novos caminhos de pesquisas.

\section{Considerações Finais}

Por fim, vale ressaltar que novas experiências como as de Belo Horizonte devem ser incentivadas pelo Ministério da Saúde, visando o delineamento de novas redes de serviços articuladas, principalmente entre a assistência social e a Psicologia e Psiquiatria no sentido de um maior cuidado com o problema da moradia de rua, entendendo esta como uma questão que pertence à saúde mental primeiramente para ser trabalhada posteriormente em redes de inclusão social (Duarte, 2007; Drake et al., 1997). Espera-se, assim, que esta pesquisa, apesar de suas limitações quanto ao número de sujeitos restrito a uma região do país, e delineamento correlacional, que não permitem inferências causais mais seguras, catalise novas investigações acerca do problema de saúde mental do morador de rua. Entre as possibilidades para estudos futuros, ressalta-se a importância de se verificar a ocorrência de funcionamento diferencial dos itens (DIF) entre as distintas amostras (por exemplo, moradores de rua e população geral), investigando se as diferenças encontradas entre as amostras refletem distintos níveis de severidade ou podem ser explicadas por outros elementos não diretamente avaliados (por exemplo, compreensão do texto, entre outros).

O acúmulo de pesquisas na área, visando demarcar este campo de atuação, pode levar ao estabelecimento de novas políticas públicas que sejam baseadas em evidências. Este tipo de sugestão também foi dada por Borysow \& Furtado (2013), ao enfatizar que a rede socioassistencial tem sido a forma de acesso ao serviço de saúde pela população de rua, deixando a sugestão de um trabalho intersetorial mais detido e bem efetivado para essas pessoas à rede pública de saúde.

Resta ainda lembrar que, embora as informações sobre os transtornos da personalidade através do IDTP sejam práticas e rápidas para 
serem obtidas e poderem ser aplicadas em um grande número de pessoas, há que se lembrar de que este não pode ser o único método utilizado na avaliação dos transtornos da personalidade. Sua função é de triagem e não como método definitivo para avaliação dos transtornos. Estes requerem um grande número de informações para entender o funcionamento da personalidade, evitando erros de diagnósticos clínicos (Carvalho, 2008). Ainda assim, com base nos dados encontrados, realçaram-se também neste estudo, para além do objetivo principal, evidências de validade com base em variáveis externas (amostra de moradores de ruas, pacientes e não pacientes) para o IDTP. É importante ressaltar que o clínico, de acordo com Millon e Davis (1996), precisa entender melhor o funcionamento da personalidade antes que sejam empregados classificaçãoes de transtornos e comorbidades, sem antes analisar minuciosamente os estilos adaptativos de funcionamento da personalidade em questão. 


\section{José Maria Montiel}

Doutor em Psicologia pela Universidade São Francisco, Itatiba- SP. Brasil. Docente do Centro Universitário Fundação Instituto de Ensino para Osasco, Osasco - SP. Brasil. E-mail: montieljm@hotmail.com

\section{Daniel Bartholomeu}

Doutor em Psicologia pela Universidade São Francisco, Itatiba - SP. Brasil. Docente do Centro Universitário Fundação Instituto de Ensino para Osasco, Osasco - SP. Brasil. E-mail: d_bartholomeu@yahoo.com.br

\section{Lucas de Francisco Carvalho}

Doutor em Psicologia pela Universidade São Francisco, Itatiba - SP. Brasil. Docente da Universidade São Francisco, Itatiba - SP. Brasil.

\section{Fernando Pessotto}

Doutorando em Psicologia pela Universidade São Francisco, Itatiba -SP. Brasil.

\section{Endereço para envio de correspondência:}

UniFIEO - Centro Universitário Fundação Instituto de Ensino para Osasco, Programa de Pós Graduação Stricto Sensu - Psicologia Educacional. Avenida Franz Voegeli $\mathrm{n}^{\circ}$ 300. Continental. CEP: 6020-190. Osasco - SP. Brasil.

Recebido 01/10/2013, $1^{\text {a }}$ Reformulação 29/07/2014, Aprovado 12/09/2014. 


\section{Referências}

American Psychiatry Association [APA]. (2013). Diagnostic and statistical manual of mental disorders 5. Washington, DC: American Psychiatry Association.

American Psychiatry Association [APA]. (2002). DSM-IV-TR. Manual diagnóstico e estatístico de transtornos mentais. (4a ed., C. Dornelles, trad.). Porto Alegre, RS: Artes Médicas.

Bartholomeu, D.; Montiel, J. M.; Silva, M. C. R.; Cozza, H. P.; \& Machado, A. A. (2013). Transtornos de personalidade e personalidade no esporte. In Carvalho, L. F.; \& Primi, R. Perspectivas em psicologia dos transtornos da personalidade: Implicações teóricas e práticas. São Paulo, SP: Casa do Psicólogo.

Bassuk, E. L., Rubin L., \& Lauriat A. (1984). Is homelessness a mental health problem? American Journal Psychiatry, 141(12), 1546-1550.

Borysow, I. C., \& Furtado, J. P. (2013). Acesso e intersetorialidade: $\mathrm{O}$ acompanhamento de pessoas em situação de rua com transtorno mental grave. Physis, 23(1), 33-50.

Botti, N. C. L. (2009). Condições de saúde da população de rua da cidade de Belo Horizonte. Cadernos Brasileiros de Saúde Mental, 1(2) 37-54.

Botti, N. C. L., Castro, C. G., Silva, M. F., Silva, A. K., Oliveira, L. C., Castro, A. C. H. O. et al. (2010). Prevalência de depressão entre homens adultos em situação de rua em Belo Horizonte. Jornal Brasileiro de Psiquiatria, 59(1) 10-16.

Carvalho, L. F. (2008). Construção e validação do inventário dimensional dos transtornos da personalidade. Dissertação de Mestrado, Programa de Pós Graduação Stricto Sensu da Universidade São Francisco, Itatiba.

Castel, R. (1998). As metamorfoses da questão social: uma crônica do salário. Petrópolis. Vozes. Recuperado em 3 de outubro de 2010, de http://libros-en-pdf.com/descargar/ as-metamorfoses-da-questão-social-4.html

Caton, C. L. M., Hasin, D., Shrout, P. E., Opler, L. A., Hirshfield, S., Dominguez, B. et al. (2000). Risk factors for homelessness among indigent urban adults with no history of psychotic illness. American Journal of Public Health, 90, 258-263.

Drake, R. E., Yovetich, N. A., Bebout, R. R., Harris, M., \& McHugo, G. J. (1997). Integrated treatment for dually diagnosed homeless adults. Journal of Nervous \& Mental Disease, 185(5), 298-305.

Fazel S., Khosla V., Doll, H. \& Geddes, J. (2008). The prevalence of mental disorders among the homeless in western countries: Systematic review and meta-regression. Analysis.PLoS Med, 5(12), 78-88

Ferreira, P. (2006). População em situação de rua: Conceitos e mensuração. Fundação João Pinheiro. In II Encontro Nacional de Produtores e Usuários de Informações Sociais, São Paulo. Recuperado em 10 de setembro de 2010, de http://www.ibge.gov. br/confest_e_confege/pesquisa_trabalhos/ arquivosPDF/L714_02.pdf

Giorgetti, C. (2006). Moradores de rua: Uma questão Social? São Paulo, SP: Educ.

Hamburger, M. E., Lilienfeld, S. O., \& Hogben, M.(1996). Psychopathy, gender, and gender roles:Implications for antisocial and histrionic personality disorders. Journal of Personality Disorders, 10(1), 41-55.

Millon, T. (2011). Disorders of personality: Introducing a DSM/ICD spectrum from normal to abnormal. Hoboken, NJ: Wiley.

Millon, T. (1999). Personality-guided therapy. Hoboken, NJ: John Wiley \& Sons, Inc.

Millon, T. (1990). Toward a new personologi: An evolutionary model. New York, NY: Wiley.

Millon, T., \& Davis, R D. (1996). An evolutionary theory of personality disorders. In. J. F. Clarking, \& M. F. Lenzenweger (Eds.), Major theories of personality disorders (pp. 221-346). New York, NY: Guilford.

Millon T., Everly, G., \& Davis, R. (1995). Cómo puede facilitarse la integratión de la psicoterapia mediante el conocimiento de la psicopotalogia? Clinica y Salud, 6(2), 109-129.

Millon, T., \& Davis, R. D. (1998). Ten subtypes of psychopathy. In T. Millon, E. Simonsen, M. Birket-Smith, \& R. D. Davis (Eds.), Psychopathy: Antisocial, criminal and violent behavior (pp. 161-170). New York, NY: Guilford. 
Millon, T., Grossman, S., Millon, C., Meagher, S., \& Ramnath, R. (2004). Personlity disorders in modern life. Hoboken, NJ: Jonh Wiley \& Sons.

Neves, D. P. (1995). A miséria em espetáculo. Revista Serviço Social e Sociedade. 47, 79-98.

Rosa, A. S., Secco, M. G., \& Brêtas, A. C. P. (2006). O cuidado em situação de rua: revendo o significado do processo saúdedoença. Revista Brasileira de Enfermagem, 59(3), 331-336.

Silva, M. L. L. (2006). Mudanças recentes do mundo do trabalho e o fenômeno população em situação de rua no Brasil 1995-2005. Dissertação de Mestrado. Universidade de Brasília, Brasília.
Skodol, A. E., Clark, L. A., Bender, D. S., Krueger, R. F., Livesley, W. J., Morey, L. C. et al. (2011). Proposed changes in personality and personality disorder assessment and diagnosis for DSM-5, Part I: Description and rationale. Personality Disorders: Theory, Research, and Treatment, 2, 4-22.

Timms P. W., \& Fry, A. H. (1989). Homelessness and mental illness. Health Trends, 21(3), 70-71.

Westen, D., \&Bradley, R.(2005).Prototypediagnosis of personality. In S. Strack (Ed.), Handbook de personology and psychopathology (pp. 238256). New York: Wiley.

Widiger, T.A. \& Clark, L.A. (2000). TowardDSM-V and the classification of psychopathology. Psychological Bulletin, 126(6), 946-963. 